\section{Measuring frailty in population-based healthcare databases: multi-dimensional prognostic indices for the improvement of geriatric care}

\author{
Janet Sultana, ${ }^{1}$ Giorgio Basile, ${ }^{1}$ \\ Gianluca Trifirò ${ }^{2}$ \\ ${ }^{1}$ Department of Clinical and Experimental \\ Medicine; ${ }^{2}$ Department of Biomedical and \\ Dental Sciences and Morphofunctional \\ Imaging, G. Martino Hospital, University \\ of Messina, Messina, Italy
}

\section{Abstract}

The prognostic evaluation of geriatric patients is critical in helping clinicians to weigh the risks versus the benefits of available therapeutic options. Frailty contributes significantly to the risk of mortality in older patients and is already known to have implications on the outcome of treatment in a clinical context. The multi-dimensional prognostic index (MPI) is a prognostic tool based on a comprehensive geriatric assessment and includes detailed information on patient cognition, functionality, disease and drug burden. The value of the MPI in predicting mortality has already been shown in hospital and community settings but never in a population-based healthcare database setting. One of the aims of the ongoing EU-funded MPI_Age project is to improve our understanding of how geriatric frailty data can be identified in healthcare databases and whether this can be used to predict serious adverse events associated with pharmacotherapy. Our findings suggest that data on functionality in elderly patients is poorly registered in The Health Improvement Network (THIN), a UK nationwide general practice database, and only few of the functionality domains could be used in a population-based analysis. The most commonly registered functionality information was related to mobility, dressing, accommodation and cognition. Our results suggest that some of these functionality domains are predictive of short- and long-term mortality in community-dwelling patients. This may have implications in observational research where frailty is an unmeasured confounder.

\section{Introduction}

The prognostic evaluation of geriatric patients is critical in helping clinicians to weigh the risks versus the benefits of available therapeutic options with the final goal of making the best clinical decision. In very old and frail patients, any pharmacological intervention may have an impact on patient survival. Frailty is characterized by an accelerated loss of functional reserves and decreased ability to maintain homeostasis, resulting in a heightened physical vulnerability and diminished capacity for recovery after a harmful event. Frailty contributes significantly to the risk of mortality in older patients and is a product of various factors such as presence and severity of co-morbidities, polypharmacy, functionality and cognitive status as well as social support. There are currently two main models of frailty: the phenotype model, ${ }^{1}$ defined as unintended weight loss, exhaustion, weakness, reduced walking speed and limited physical activity, and the cumulative deficit model, ${ }^{2}$ in which the presence of disease and disability/functionality as deficits are considered together. A systematic review ${ }^{3}$ of studies investigating frailty found that the overall prevalence of frailty in community-dwelling adults aged 65 years and older varies significantly, ranging from $4.0 \%$ to $59.1 \%$, with an overall adjusted prevalence of $10 \%$ in 21 studies enrolling 61,500 patients. Collard et al. also found that frailty increased with age, as would be expected, but also that it was higher among women than men.

\section{Materials and Methods}

\section{The prognostic value of frailty}

Frailty is already known to have implications on the outcome in a clinical context. Physicians take patient's frailty into account when deciding on treatment, for example avoiding interventions that are associated to a high risk of serious adverse events, discontinuing drugs aimed at primary prevention (e.g., aspirin or statins) in very old patients. This distinction between elderly patients who are more or less frail in the clinical decision to treat and how to treat patients results in the differential treatment of patients, particularly in the oldest old. Since information on patient frailty is not commonly included in post-marketing observational studies investigating drug safety in older persons, results from these studies are necessarily subject to a degree of unmeasured confounding, specifically confounding by frailty. This constitutes a problem insofar as there is less evidence on the frail elderly patients who are most at risk, or conversely who may benefit most, from a given therapeutic intervention. There are several standardized methods that were developed to measure frailty and subsequently predict clinical outcomes. A systematic review ${ }^{4}$ published in 2012 identified a total of 16 studies on prognostic indices in older patients, of which only 5 included the evaluation of functionality
Correspondence: Gianluca Trifirò, Department of Biomedical and Dental Sciences and Morphofunctional Imaging, G. Martino Hospital, University of Messina, via Consolare Valeria 1, 98125 , Messina, Italy.

Tel.: +39.0902213264 - Fax: +39.0902213300.

E-mail: trifirog@unime.it

Key words: Frailty; elderly; prognostic indicators population-based databases; The Health Improvement Network.

Funding: this paper describes some of the work carried out within the MPIAge project. The MPI_Age project was funded by the European Commission under the European Commission Public Health Programme 2008-2013.

Contributions: JS, GB and GT wrote and revised the manuscript; GT conceived the idea for the manuscript.

Conflicts of interest: the authors have no conflicts of interest to declare.

Received for publication: 20 October 2015. Revision received: 30 December 2015.

Accepted for publication: 20 January 2016.

This work is licensed under a Creative Commons Attribution-NonCommercial 4.0 International License (CC BY-NC 4.0)

CC Copyright J. Sultana et al., 2016

Licensee PAGEPress, Italy

Geriatric Care 2016; 2:5596

doi:10.4081/gc.2016.5596

domains in community-dwelling patients; of these 5, only one was carried out in Europe. ${ }^{5}$ Another systematic review included 7 studies published between 2011 and 2012 on the predictive value of functionality domains in relation to adverse events of cancer drugs. ${ }^{6}$ However, there do not appear to be any other studies investigating how a prognostic index could predict adverse events due to drug therapy. The role of frailty as a prognostic factor, especially frailty with the inclusion of functionality domains, appears to be somewhat under-valued in the study of geriatric health outcomes, in particular as a predictor of risk of adverse events in elderly persons receiving pharmacological treatment. One of the aims of the currently ongoing EUfunded MPI_Age research project ${ }^{7}$ is to improve our understanding of how data on frailty of geriatric outpatients at population level can be identified in healthcare databases and whether this can be used to predict serious adverse events associated with pharmacotherapy.

\section{Use of the multi-dimensional} prognostic index in clinical research

The multi-dimensional prognostic index (MPI) is a prognostic index developed in Italy based on a standardized comprehensive geriatric assessment (CGA) that provides informa- 
tion on functional, cognitive and nutritional status, co-morbidities, medications prescribed and social support. The MPI was found to have a positive predictive value at one year and one month in a cohort of in-patients aged $\geq 65$ admitted to the Geriatric Unit of Research Hospital from Southern Italy from January 1 to December 31, 2004 ( $\mathrm{N}=383)^{8}$. The MPI was later also applied in a much larger cohort of community-dwelling patients aged $\geq 65(\mathrm{~N}=12,020)$ in Veneto region using an administrative claims database. ${ }^{9}$ In this study, patients were enrolled if they required a healthcare intervention reimbursable by the Italian National Health Service for which a standardized CGA was assessed. However, the standardized CGA is mainly registered if elderly persons request admission to homecare or request home-care services, making the sample of elderly persons not fully representative of community-dwelling elderly adults. The MPI has also been used in another observational study within the same catchment area to investigate whether the protective effect of statins on mortality is also seen in frail elderly persons diagnosed with diabetes..$^{10}$ Findings from this study suggest that statins continue to have a protective effect even in the frailest elderly persons, although these drugs are more commonly prescribed in healthier elderly persons. Nevertheless, this study is also limited to those persons seeking nursing home admission or home-care services, reducing the generalizability of this study. The MPI has more recently been applied in a population-based cohort of 2472 elderly patients from the Swedish National Study on Aging and Care. Higher MPI scores were found to be associated with longer hospital stay and with a shorter survival time. ${ }^{11}$ In this study, the MPI was calculated using seven domains: PADL, I-ADL, cognitive function (mini-mental state exam), illness severity and comorbidity, the number of medications, and living arrangement. Each of the domains were scored 0 to denote low risk of mortality and/or longer hospitalization, 0.5 to denote a moderate risk, and 1 denoting a high risk. In this study for the first time the MPI was validated in a populationbased setting and in subjects with a very long (10 to 12 years) follow-up period. Using MPI values and grades of severity it was possible to predict life expectancy in terms of survival years in individual subjects.

\section{Results}

\section{Measuring frailty to predict mortality in population-based general practice databases}

Population-based general practice databases containing data on a large number of elderly persons are potentially avaluable source to capture frailty-related information on a more heterogeneous group of elderly patients at population-based level. The value of an MPI-like prognostic index derived from a general practitioner (GP) database would be significant, as it allows the evaluation of the risk-benefit profile of available and currently used medication in elderly out-patients of all health statuses within the general population. Within the funded MPI_Age project, our first objective was to explore if and how commonly any information on MPI-related functionality domainswere registered in a GP database. It is already known that demographic information as well as health indicators such as co-morbidities and prescription data are registered within GP databases and have been frequently used to adjust risk estimates of the risks or benefits associated with drug use. However, much less is known about the registration of functionality domains such as cognitive status and activities of daily living (eating, bathing, dressing, toileting and walking with a defined level of independence as well as continence). Our second objective was to investigate the prognostic value of the measured and registered functionality domains, in addition to co-morbidities and concomitantly prescribed medications, in the prediction of death in a cohort of elderly persons ( $\geq 65$ years) identified from a GP database. Exploratory work carried out in The Health Improvement Network, also known as The Health Improvement Network (THIN) (a UK nationwide GP database) shows that this database does not contain all the information to calculate MPI score as had been used in previous cohort studies conducted in hospital or in outpatient setting using administrative databases. In order to identify functionalityrelated domains, the GP database was screened for GP-registered functionality-related codes matching key words related to the MPI functionality domains, i.e., the activities of daily living, accommodation (nursing home or other residence), nursing needs (tracheostomy, catheterization, etc.), mobility and so on. Once identified, the individual functionality-related codes were grouped into functionality domains, the usefulness of which depends largely on how frequently the functionality information is available and in how many patients it is available. Our findings suggest that information on functionality in elderly patients is poorly registered and few of the functionality domains could be used in a population-based analysis. The most commonly registered functionality information was related to mobility, dressing, accommodation and cognition. In order to understand the predictive value of the functionality domains that were identified, we compared the predictive value of the following Cox regression models including the following covariates: age and sex, age, sex and co-morbidities and the last model with age, sex, co-morbidities and a functionality domain to see whether the prediction of 1month and 1-year mortality in communitydwelling persons $\geq 65$ improved with the inclusion of the functionality domains. Our results suggest that some of the identified functionality domains registered in a GP databases are predictive of short- and long-term mortality in community-dwelling patients.

\section{Discussion}

\section{Advantages of population-based healthcare databases in frailty research}

The additional value of a population-based MPI containing data on patient functionality is that health-related outcomes in these patients can be adjusted for functionality as well healthrelated factors, thus removing functionalityrelated confounding. ${ }^{12}$ This can shed light on which patients are more vulnerable overall, thus helping clinicians in assessing the risk and benefit of therapeutic options available to them and helping healthcare providers/regulators more accurately identify trends in clinical outcomes that favor a therapeutic approach or otherwise. There are several advantages of population-based databases as a source of clinical data. They often contain data on large populations, which in some cases is representative of a whole country or Region. The patients enrolled have a variety of clinical characteristics as seen in the general population, including those who would be excluded from clinical trials, such as elderly persons with a high morbidity burden and polypharmacy. In addition, these databases often have data recorded over many years, allowing long follow-up periods. Finally, they contain information from a real-world clinical setting rather than a simpler but relatively artificial setting such as a clinical trial. Various population-based databases have been used to conduct observational studies investigating clinical outcomes using demographic patient data, drug prescriptions/hospital procedures/hospitalization and co-morbidities, especially those associated with pharmacotherapy and drug safety. Such studies include, but are not limited to, investigation of all-cause mortality associated with drugs ${ }^{13,14}$ and the risk of drug-associated hospitalization for hip fracture, atrial fibrillation ${ }^{15}$ or pneumonia. ${ }^{16} \mathrm{~A}$ specific example of the relevance of observational studies in the past ten years is the theme of antipsychotic safety in elderly persons, which has been extensively investigated using population-based databases. ${ }^{17}$ Despite concerns about the limitations of observational studies compared to randomized controlled trials, a recent Cochrane meta-analysis reported that finding 
derived from real world observational studies are comparable to results obtained from randomized controlled trials, regardless of specific observational study design, heterogeneity, or inclusion of studies of pharmacological interventions. ${ }^{18}$ Observational studies investigating drug safety produce risk estimates that can be adjusted for demographic factors (age and sex) as well as clinical characteristics, in order to account for their effect on the risk estimate, but have rarely, if at all, been adjusted for frailty.

\section{Conclusions}

Prognostic indexes such as MPI can be helpful for guiding physicians in choosing the pharmacological treatment with the best benefitrisk profile in very old and frail patients. Findings from a nationwide GP database suggest that functionality information is not systematically registered for the whole elderly population but, whenever registered, some functionality domains are shown to be predictive of both short- and long-term mortality in geriatric patients. Proactive action should be taken to increase the evaluation of functionality domains in elderly persons and the registration of their functionality status in general practice setting, as this information can be useful both to clinicians and healthcare providers for optimizing drug use in older people.

\section{References}

1. Fried LP, Tangen CM, Walston J, et al. Frailty in older adults: evidence for a phenotype. J Gerontol A Biol Sci Med Sci 2001;56:M146-56.

2. Rockwood K, Mitnitski A. Frailty in rela- tion to the accumulation of deficits. J Gerontol A Biol Sci Med Sci 2007;62:722-7.

3. Collard RM, Boter H, Schoevers RA, Oude Voshaar RC. Prevalence of frailty in community-dwelling older persons: a systematic review. J Am Geriatr Soc 2012;60: 1487-92.

4. Yourman LC, Lee SJ, Schonberg MA, et al. Prognostic indices for older adults: a systematic review. JAMA 2012;307:182e192.

5. Mazzaglia G, Roti L, Corsini G, et al. Screening of older community-dwelling people at risk for death and hospitalization: the Assistenza Socio-Sanitaria in Italia project. J Am Geriatr Soc 2007;55: 1955-60.

6. Puts MT, Santos B, Hardt J, et al. An update on a systematic review of the use of geriatric assessment for older adults in oncology. Ann Oncol 2014;25:307-15.

7. Pilotto A, Sancarlo D, Polidori MC, et al. on behalf of the MPI_AGE Project Investigators. The MPI_AGE European Project: Using Multidimensional Prognostic Indices (MPI) to improve cost-effectiveness of interventions in multimorbid frail older persons. Background, aim and design. Eur Geriatr Med 2015;6:184-8.

8. Pilotto A, Ferrucci L, Franceschi M, et al. Development and validation of a multidimensional prognostic index for one-year mortality from comprehensive geriatric assessment in hospitalized older patients. Rejuvenation Res 2008;11:151-61.

9. Pilotto A, Gallina P, Fontana A, et al. Development and validation of a Multidimensional Prognostic Index for mortality based on a standardized Multidimensional Assessment Schedule (MPI-SVaMA) in community-dwelling older subjects. J Am Med Dir Assoc 2013;14:287-92.

10. Pilotto A, Panza F, Copetti M, et al. Statin treatment and mortality in communitydwelling frail older patients with diabetes mellitus: a retrospective observational study. PLoS One 2015;10:e0130946.

11. Angleman SB, Santoni G, Pilotto A, et al. Multidimensional Prognostic Index in Association with Future Mortality and Number of Hospital Days in a PopulationBased Sample of Older Adults: results of the EU Funded MPI_AGE Project. PLoS One 2015;10:e0133789.

12. Kim DH, Schneeweiss S. Measuring frailty using claims data for pharmacoepidemiologic studies of mortality in older adults: evidence and recommendations. Pharma-coepidemiol Drug Saf 2014;23: 891-901.

13. Gerhard T, Huybrechts K, Olfson M, et al. Comparative mortality risks of antipsychotic medications in community-dwelling older adults. Br J Psychiatry 2014;205:44-51.

14. Trifirò G, Verhamme KM, Ziere G, et al. Allcause mortality associated with atypical and typical antipsychotics in demented outpatients. Pharmacoepidemiol Drug Saf 2007;16:538-44.

15. Herrera L, Leal I, Lapi F, et al. Risk of atrial fibrillation among bisphosphonate users: a multicenter, population-based, Italian study. Osteoporos Int 2015;26: 1499-506.

16. Sterrantino C, Trifirò G, Lapi F, et al. Burden of community-acquired pneumonia in Italian general practice. Eur Respir J 2013;42:1739-42.

17. Sultana J, Spina E, Trifirò G. Antidepressant use in the elderly: the role of pharmacodynamics and pharmacokinetics in drug safety. Expert Opin Drug Metab Toxicol 2015;11:883-92.

18. Anglemyer A, Horvath HT, Bero L. Healthcare outcomes assessed with observational study designs compared with those assessed in randomized trials. Cochrane Database Syst Rev 2014;4: MR000034. 